\title{
Time-released garlic powder tablets lower systolic and diastolic blood pressure in men with mild and moderate arterial hypertension
}

\author{
Igor A Sobenin ${ }^{1,4}$, Irina V Andrianova ${ }^{2,4}$, Igor V Fomchenkov ${ }^{3}$, Tatiana V Gorchakova ${ }^{1,2}$ \\ and Alexander N Orekhov ${ }^{1,4}$
}

Numerous clinical investigations that have focused on the hypotensive effects of garlic-based preparations have led to controversial results that may be partially because of differences in the composition of the preparations and in the biological responses they induce. It is possible that garlic powder tablets with a prolonged mode of action could induce more potent biological effects. In this double-blind, placebo-controlled trial with an active control arm, the hypotensive action of time-released garlic powder tablets (Allicor) was compared with that of regular garlic pills (Kwai) in 84 men with mild or moderate arterial hypertension. After an 8-week placebo treatment run-in phase, patients were randomized either to $600 \mathrm{mg}$ Allicor $(n=30)$ or to placebo $(n=20)$ daily for 8 weeks. In addition, in the open-label branch, patients received either $2400 \mathrm{mg}$ Allicor daily $(n=18)$ or $900 \mathrm{mg}$ Kwai daily $(n=16)$. Allicor treatment $(600 \mathrm{mg}$ daily) resulted in a reduction of both systolic and diastolic blood pressures by $7.0 \mathrm{~mm} \mathrm{Hg}(95 \%$ confidence interval $(95 \% \mathrm{Cl}): 5.3-8.7)$ and $3.8 \mathrm{~mm} \mathrm{Hg}(95 \% \mathrm{Cl}: 2.7-4.8)$, respectively. Increasing the Allicor dosage to $2400 \mathrm{mg}$ daily did not provide any additional benefit. Treatment with Kwai resulted in the same decrease in systolic blood pressure $(5.4 \mathrm{~mm} \mathrm{Hg}, 95 \% \mathrm{Cl}: 1.9-8.8)$ as that seen with Allicor, but no decrease in diastolic blood pressure was observed with Kwai. Different effects of Allicor and Kwai on diastolic blood pressure may be because of the prolonged action of Allicor, which allows better bioavailability of the vasoactive constituents of garlic powder. The results of this study show that time-released garlic powder tablets are more effective for the treatment of mild and moderate arterial hypertension than are regular garlic supplements.

Hypertension Research (2009) 32, 433-437; doi:10.1038/hr.2009.36; published online 24 April 2009

Keywords: arterial hypertension; garlic; placebo-controlled trial

\section{INTRODUCTION}

The medicinal use of garlic (Allium sativum) dates back thousands of years, but there was little scientific support of its therapeutic and pharmacological properties until recently, with the extensive evaluation of the anti-atherosclerotic and cardiovascular-protective effects of raw garlic and garlic-based dietary supplements. The results of numerous clinical investigations have shown that garlic may produce beneficial effects on different cardiovascular risk factors: garlic is known to reduce serum cholesterol levels in humans, inhibit cholesterol biosynthesis, lower arterial blood pressure, suppress low-density lipoprotein oxidation, lower plasma fibrinogen and increase fibrinolytic activity, thus possessing anti-atherosclerotic properties. ${ }^{1-6}$ Among all risk factors for atherosclerosis, arterial hypertension, along with dyslipidemia, is thought to be the most potent factor that greatly increases the risk of cardiovascular diseases. ${ }^{7,8}$ Clinical investigations that focused on the effect of garlic in arterial hypertension have revealed its moderate hypotensive action in most studies; ${ }^{9-13}$ however, controversial data exist. ${ }^{14}$ These discrepancies may be because of several factors, including a lack of consistency among studies in relation to dosage, standardization of garlic preparations and period of treatment. Not all garlic preparations may be assumed equivalent in their composition and, more importantly, in the biological response they precipitate. The recently developed garlic powder-based dietary supplement, Allicor, is characterized by a prolonged mode of action that differentiates it from other products on the market. We have hypothesized that time-released garlic powder tablets may provide more potent pharmacological effects. To test this hypothesis, we carried out a double-blind, placebo- and active-controlled study of hypotensive action of Allicor

${ }^{1}$ Institute of General Pathology and Pathophysiology, Russian Academy of Medical Sciences, Moscow, Russia; ${ }^{2}$ Russian Cardiology Research Center, Institute of Experimental Cardiology, Moscow, Russia; ${ }^{3}$ Russian State Medical University, Moscow, Russia and ${ }^{4}$ Institute for Atherosclerosis Research, Russian Academy of Natural Sciences, Moscow, Russia

Correspondence: Dr TV Gorchakova, Russian Cardiology Research Center, Institute of Experimental Cardiology, 15-a 3rd Cherepkovskaya Str., Moscow 121552, Russia. E-mail: t-gorchakova@mail.ru

Received 8 September 2008; revised 16 December 2008; accepted 10 February 2009; published online 24 April 2009 
in comparison with regular garlic powder tablets (Kwai) in men with mild and moderate arterial hypertension.

\section{METHODS}

\section{Subjects and study design}

This study was a randomized, placebo-controlled, double-masked outpatient clinical trial with an active control arm carried out in 84 men (35-70 years old) with newly diagnosed mild and moderate arterial hypertension. The study was carried out in accordance with the Declaration of Helsinki Principles of 1975 and the revisions from 1983. This study was approved by the institutional review board at the Institute for Atherosclerosis Research for the protection of human subjects in research. All participants gave their informed consent before their inclusion in the study. The included participants had no diseases demanding continuous administration of cardiotropic, sugar-lowering drugs or diuretics. The patients who were admitted had a systolic blood pressure (SBP) between 150 and $160 \mathrm{~mm} \mathrm{Hg}$ and a diastolic blood pressure (DBP) between 90 and $115 \mathrm{~mm} \mathrm{Hg}$ after an 8-week placebo-treated acclimatization phase. The study participants were either randomly switched to $600 \mathrm{mg}$ Allicor daily (INAT-Farma, Moscow, Russia) (one tablet containing $300 \mathrm{mg}$ garlic powder twice a day, $n=30$ ) or continued to receive a placebo of identical appearance $(n=20)$. Some patients were randomly switched to the open-label branch of the study and received either $2400 \mathrm{mg}$ Allicor daily (two tablets four times a day, $n=18$ ) or $900 \mathrm{mg}$ Kwai (Lichtwer Pharma GmbH, West Berlin, Germany) daily (one tablet containing $300 \mathrm{mg}$ garlic powder three times a day, $n=16$ ). Randomization was carried out with the use of a random number generator. The treatment period in all groups lasted for 8 weeks. The baseline data on clinical and demographic characteristics of study participants are presented in Table 1. All participants received similar dietary and behavioral recommendations and consumed a low-salt diet.

\section{Blood pressure measurements}

Arterial blood pressure was measured at the time of inclusion and every 4 weeks thereafter for 16 weeks of the study. Blood pressure measurement was always performed in the morning on the right and left arms, in supine, sitting and standing positions. The results of the second and the third measurements were recorded, and the mean value of a total of 12 measurements was used as an integral estimate of arterial blood pressure.

\section{Statistical analysis}

Data were processed using the SPSS 10.1.7 statistical program package (SPSS, Chicago, IL, USA). After examination of the variable distribution, the MannWhitney statistics were used for between-group comparisons, the Wilcoxon statistics were used for within-group effect assessments, and one-way ANOVA (analysis of variance) was used for comparisons of the treatment effects between groups. Results are expressed in terms of means, s.e.m. and 95\% CI
( $95 \%$ confidence interval), if applicable. Significance was defined at the 0.05 level of confidence.

\section{RESULTS}

Subject enrollment and compliance

A total of 90 participants were included in the study. During the treatment period, six patients discontinued study medications, one in the Allicor (2400 mg daily) group and one in the Kwai group, because of gastrointestinal complaints, and four at their own request (one in the Allicor (2400 mg daily) group and three in the Kwai group). None of the patients discontinued study medications in the Allicor $(600 \mathrm{mg}$ daily) group nor in the placebo group. There were no side effects from Allicor, including gastrointestinal complaints. Thus, 84 participants were evaluated at the end of the 8 -week treatment period (30 in the $600 \mathrm{mg}$ Allicor group; 18 in the $2400 \mathrm{mg}$ Allicor group; 20 in the placebo group; and 16 in the Kwai group).

\section{Baseline characteristics}

Table 1 summarizes the baseline characteristics of study participants according to their treatment assignments. The groups did not differ significantly in demographic and clinical characteristics either at the time of inclusion or after the 8-week placebo-treated run-in phase. Thus, subsequent analyses of changes in SBP and DBP were conducted without using additional parameters as covariates.

\section{SBP}

During the 8-week placebo-treated run-in phase of the study, SBP in the total group was lowered by $3.2 \pm 0.7 \mathrm{~mm} \mathrm{Hg}$ (95\% CI: $1.7-4.6$, $P<0.001)$. Further dynamics of SBP are shown in Figure 1.

In the patients who received $600 \mathrm{mg}$ Allicor daily, SBP was lowered by $5.5 \pm 0.8 \mathrm{~mm} \mathrm{Hg}(95 \% \mathrm{CI}: 3.8-7.2, P<0.001)$ after 4 weeks and by $7.0 \pm 0.8 \mathrm{~mm} \mathrm{Hg}$ (95\% CI: 5.3-8.7, $P<0.001$ ) after 8 weeks of treatment (Figure 1). The difference in SBP changes between Allicortreated and placebo groups was statistically significant after 4 and 8 weeks of treatment $(P<0.001)$.

Treatment with $2400 \mathrm{mg}$ Allicor daily also resulted in a statistically significant reduction in SBP. After 4 weeks of treatment, SBP was lowered by $5.4 \pm 0.5 \mathrm{~mm} \mathrm{Hg}$ (95\% CI: 4.4-6.5, $P<0.001$ ), and after 8 weeks it was lowered by $9.3 \pm 0.7 \mathrm{~mm} \mathrm{Hg}$ (95\% CI: $7.8-10.9$, $P<0.001$ ) (Figure 1). The difference in SBP changes between Allicor-treated and placebo groups was statistically significant after 4 and 8 weeks of treatment $(P<0.001)$. The hypotensive effect of $2400 \mathrm{mg}$ Allicor daily did not differ significantly from that of the $600 \mathrm{mg}$ daily group $(P=0.092)$.

Table 1 Baseline demographic and clinical characteristics of study participants

\begin{tabular}{|c|c|c|c|c|}
\hline Variable & Allicor (600 mg daily) & Allicor (2400 mg daily) & Kwai (900 mg daily) & Placebo \\
\hline Number of patients, $n$ & 30 & 18 & 16 & 20 \\
\hline Age, years & $51.5 \pm 2.1(47.2-55.9)$ & $50.9 \pm 2.4(45.8-55.9)$ & $52.2 \pm 2.5(46.9-57.5)$ & $52.7 \pm 2.5(47.4-58.0)$ \\
\hline Alcohol consumption, $n(\%)$ & $12(40.0)$ & $7(38.9)$ & $7(43.8)$ & $8(40.0)$ \\
\hline Smoking history, $n(\%)$ & $12(40.0)$ & $7(38.9)$ & $7(43.8)$ & $8(40.0)$ \\
\hline Family history of hypertension, $n(\%)$ & $3(10.0)$ & $2(11.1)$ & $2(12.5)$ & $2(10.0)$ \\
\hline Cardiovascular history, $n(\%)$ & $6(20.0)$ & $4(22.2)$ & $4(25.0)$ & $4(20.0)$ \\
\hline Body mass index, $\mathrm{kg} \mathrm{m}^{-2}$ & $25.3 \pm 0.6(24.0-26.5)$ & $25.5 \pm 0.8(23.9-27.1)$ & $25.3 \pm 0.8(23.7-26.9)$ & $26.0 \pm 1.0(23.8-28.1)$ \\
\hline $\mathrm{SBP}, \mathrm{mm} \mathrm{Hg}$ at the inclusion to the study & $158.0 \pm 2.8(152.4-163.6)$ & $154.0 \pm 3.4(146.9-161.1)$ & $156.9 \pm 2.8(151.0-162.8)$ & $154.8 \pm 3.2(148.1-161.5)$ \\
\hline After run-in phase & $155.6 \pm 2.5(150.5-160.7)$ & $153.1 \pm 2.7(147.3-158.9)$ & $152.1 \pm 2.7(146.3 \pm 157.9)$ & $149.8 \pm 2.8(143.9-155.7)$ \\
\hline $\mathrm{DBP}, \mathrm{mm} \mathrm{Hg}$ at the inclusion to the study & $97.0 \pm 1.0(94.9-99.1)$ & $95.0 \pm 0.6(93.6-96.4)$ & $97.5 \pm 1.0(95.4-99.6)$ & $96.1 \pm 1.3(93.4-98.9)$ \\
\hline After run-in phase & $96.0 \pm 0.9(94.2-97.9)$ & $94.6 \pm 0.5(93.5-95.8)$ & $96.4 \pm 0.8(94.7-98.2)$ & $94.4 \pm 1.4(91.5-97.2)$ \\
\hline
\end{tabular}

Abbreviations: DBP, diastolic blood pressure; SBP, systolic blood pressure.

Data for age, body mass index and blood pressure are presented as means \pm s.e.m. and $95 \% \mathrm{Cl}$ given in parentheses. 


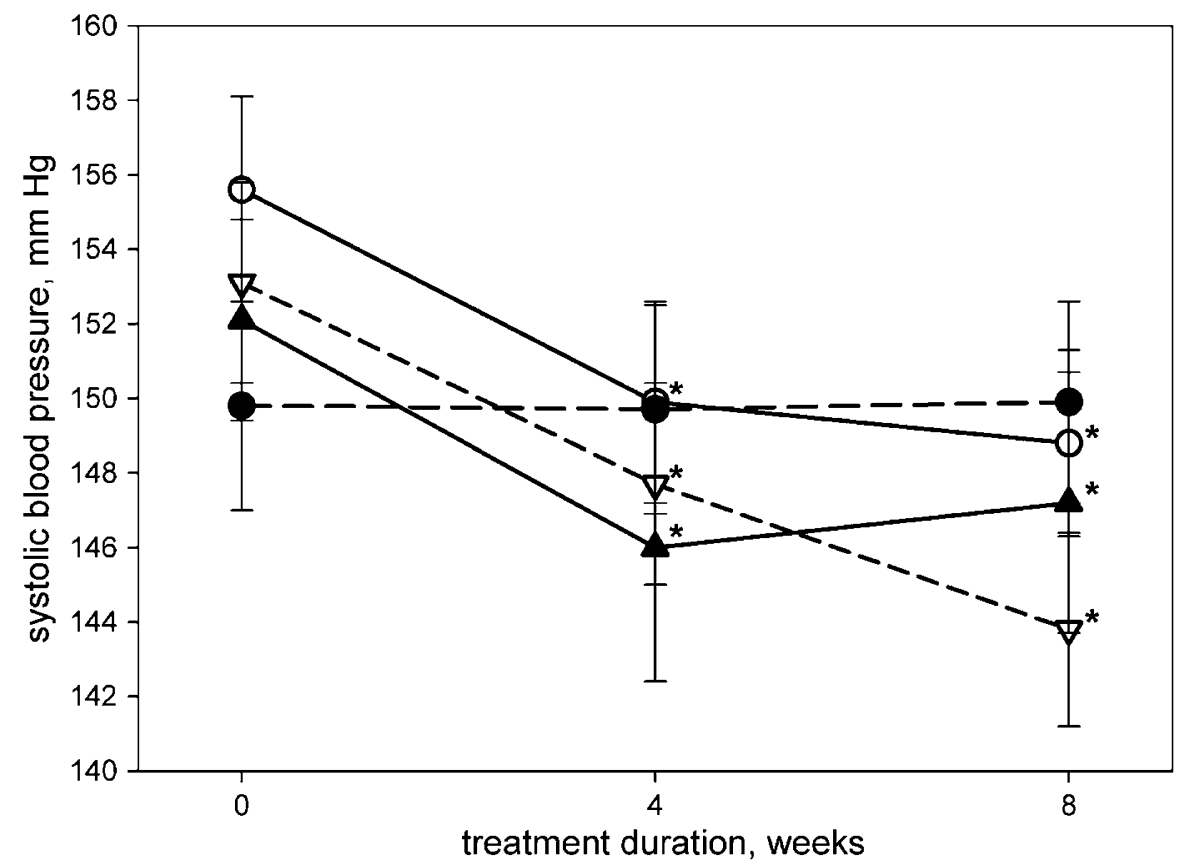

Figure 1 The dynamics of systolic blood pressure. Open circles/solid line, $600 \mathrm{mg}$ Allicor daily; solid circles/long dash, placebo; open triangles/short dash, $2400 \mathrm{mg}$ Allicor daily; solid triangles/solid line, $900 \mathrm{mg}$ Kwai daily. Asterisk indicates a significant difference from the beginning of the treatment phase; $P<0.05$, Wilcoxon signed-rank test.

In patients who received $900 \mathrm{mg}$ Kwai daily, SBP was lowered by $5.3 \pm 1.5 \mathrm{~mm} \mathrm{Hg}(95 \%$ CI: $2.1-8.6)$ after 4 weeks of treatment $(P=0.006)$, and the same effect was observed at the end of the study (SBP decreased by $5.4 \pm 1.6 \mathrm{~mm} \mathrm{Hg}, 95 \%$ CI: $1.9-8.8, P=0.010$ ) (Figure 1). The difference in SBP changes between Kwai-treated and placebo groups was statistically significant after 4 and 8 weeks of treatment $(P=0.009$ and $P=0.011$, respectively). The effect of $900 \mathrm{mg}$ Kwai daily on SBP did not differ significantly from that of 600 or $2400 \mathrm{mg}$ Allicor daily.

\section{DBP}

During the 8-week placebo-treated run-in phase of the study, DBP in the total group was lowered by $1.0 \pm 0.4 \mathrm{~mm} \mathrm{Hg}$ (95\% CI: $0.3-1.7$, $P<0.001)$. Further dynamics of DBP are shown in Figure 2.

In those patients who received $600 \mathrm{mg}$ Allicor daily, DBP was lowered by $2.5 \pm 0.6 \mathrm{~mm} \mathrm{Hg}$ (95\% CI: $1.1-3.8, P<0.001)$ after 4 weeks. By the end of the study, DBP decreased by $3.8 \pm 0.5 \mathrm{~mm} \mathrm{Hg}$ (95\% CI: 2.7-4.8, $P<0.001$ ) (Figure 2). The difference between DBP changes in Allicor and placebo groups was statistically significant $(P<0.001)$.

The treatment with $2400 \mathrm{mg}$ Allicor daily also resulted in a statistically significant reduction in DBP. After 4 weeks of treatment, DBP was lowered by $2.0 \pm 0.4 \mathrm{~mm} \mathrm{Hg}$ ( $95 \% \mathrm{CI}: 1.2,2.8, P<0.001)$, and after 8 weeks it was lowered by $3.2 \pm 0.5 \mathrm{~mm} \mathrm{Hg}$ (95\% CI: $2.2-4.2$, $P<0.001$ ) (Figure 2). The difference in DBP changes between Allicor-treated and placebo groups was statistically significant after 4 and 8 weeks of treatment $(P<0.001)$. There was no statistically significant difference between the effects of 600 and $2400 \mathrm{mg}$ Allicor daily on DBP.

Treatment with $900 \mathrm{mg}$ Kwai daily did not result in a statistically significant decrease in DBP compared with placebo. After 4 weeks of treatment, DBP changed by $-0.9 \pm 1.1 \mathrm{~mm} \mathrm{Hg}$ ( $95 \%$ CI from -3.2 to $1.4, P=0.156)$, and after 8 weeks it changed by $-1.0 \pm 1.2 \mathrm{~mm} \mathrm{Hg}$
(95\% CI from -3.6 to $1.5, P=0.088$ ) (Figure 2). The difference in DBP changes between Kwai-treated patients and placebo recipients was observed after 4 weeks $(P=0.046)$, but not after 8 weeks of treatment $(P=0.179)$.

\section{DISCUSSION}

The results of this study have shown that the garlic-based dietary supplement, Allicor, produces a statistically significant hypotensive effect on both SBP and DBP in men with mild and moderate arterial hypertension. These data are similar to the results from earlier studies that showed the hypotensive effects of garlic products. ${ }^{9-14}$ However, most earlier studies were non-randomized and non-placebocontrolled. Thus, the results of this randomized, placebo-controlled, double-masked clinical trial provide substantial evidence for the hypotensive effectiveness of garlic.

Valid comparisons of the hypotensive effects of two different garlicbased preparations, namely Allicor and Kwai, are made possible from this study. Both Allicor and Kwai induced moderate, but statistically significant, decreases in SBP that developed within the first 4 weeks of treatment, and garlic administration for an additional 4 weeks did not result in any additional hypotensive action. The effects of Kwai and Allicor on SBP did not differ significantly, although the dosages recommended by the manufacturers differed ( 900 and $600 \mathrm{mg}$ per day, respectively). Thus, the maximum possible hypotensive effect of garlic was achieved at the $600 \mathrm{mg}$ per day dose, and further increases in dosage did not provide additional benefits. In support of this idea, the four-fold increase in Allicor dosage to $2400 \mathrm{mg}$ per day did not produce more prominent hypotensive effects on either SBP or DBP. Taken together, these findings show that the mechanisms of hypotensive action of garlic-based preparations may be quite different from those of conventional pharmacological agents used in the treatment of arterial hypertension and may be referred to complex biological regulation of blood pressure. 


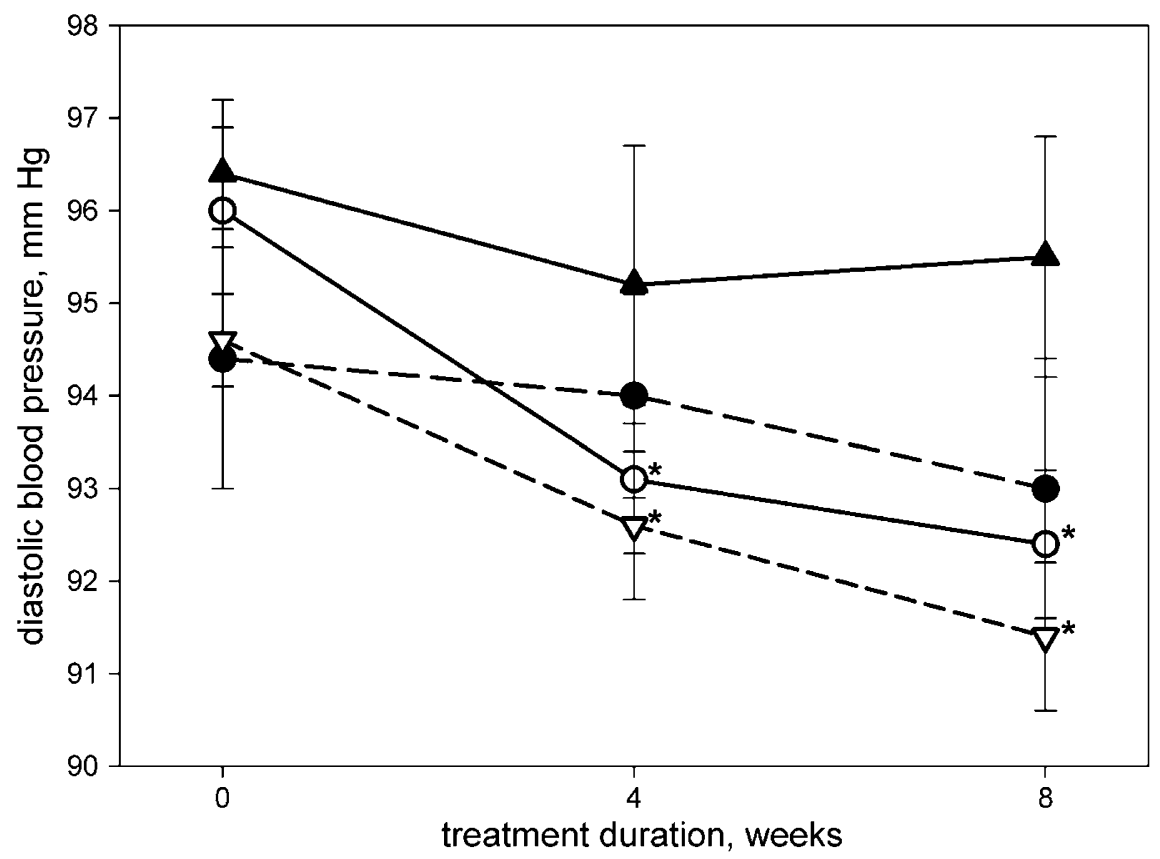

Figure 2 The dynamics of diastolic blood pressure. Open circles/solid line, $600 \mathrm{mg}$ Allicor daily; solid circles/long dash, placebo; open triangles/short dash, $2400 \mathrm{mg}$ Allicor daily; solid triangles/solid line, $900 \mathrm{mg}$ Kwai daily. Asterisk indicates a significant difference from the beginning of the treatment phase; $P<0.05$, Wilcoxon signed-rank test.

The mechanisms of hypotensive action of garlic-based preparations and garlic components remain rather obscure. Results from several animal studies show that garlic constituents are able to decrease blood pressure in hypertensive animals by producing vasodilating effects at the level of the arterial wall. ${ }^{15-27}$ Although allicin, an active ingredient released from garlic that is thought to be a systemic vasodilator, does not alter the activity of vascular prostacyclin synthase, it dilates the mesenteric circulation in cats independent of prostaglandin release or a $\beta$-adrenergic mechanism. ${ }^{16}$ In terms of relative vasodilator activity, allicin was 100 -fold less potent than sodium nitroprusside and many orders of magnitude less potent than isoproterenol; however, it significantly diminished the pulmonary pressor response to ventilatory hypoxia in isolated perfused rat lung. In addition, pulmonary vasodilator responses to allicin were independent of the synthesis of endothelial-derived relaxing factor or the activation of soluble guanylate cyclase. ${ }^{18}$ Garlic can prevent chronic inhibition of nitric oxide synthesis by L-NAME ( $N$-omega-nitro-L-arginine methyl ester) in rats, thus preventing L-NAME-induced arterial hypertension. ${ }^{24}$ In rats, garlic completely inhibited acute hypoxic pulmonary vaso constriction and induced significant dose-dependent vasorelaxation in both endothelium-intact and mechanically endothelium-disrupted pulmonary arterial rings, thus showing a combination of endothelium-dependent and endothelium-independent mechanisms for the development of the hypotensive effect. ${ }^{23}$ It was also shown that garlic juice possesses a direct relaxing effect on the smooth muscles of the aorta, trachea, intestines and isolated rabbit hearts in vitro. ${ }^{28}$ Garlic may provide a depressing effect on automaticity and tension development in the isolated rat heart, suggesting a $\beta$-adrenoreceptorblocking action. ${ }^{17}$ The hypotensive effects of garlic constituents may also be explained in part by a significant biphasic diuretic and natriuretic response and by an inhibitory dose-dependent effect on the kidney Na, K-ATPase. ${ }^{29-31}$ Finally, aqueous garlic extract, as well as allicin and ajoene, can open potassium ion channels, causing membrane hyperpolarization that closes about $20 \%$ of the L-type $\mathrm{Ca}^{2+}$ channels, the consequence of which is vasodilatation. ${ }^{32,33}$

It is necessary to note that in our study, Allicor, but not Kwai, induced a statistically significant decrease in DBP. The inconsistencies in results obtained in different studies are often explained by differences in components used during supplement preparation, the quantity of the preparation provided, the duration of the study and the influence of the manufacturing process on the composition of the garlic product; moreover, biological responses to different preparations may vary greatly. Garlic contains various organosulfur compounds, amino acids, vitamins and minerals. ${ }^{34}$ Sulfur-containing compounds such as allicin, ajoene and cicletanine and various sulfoxides may be responsible for the hypotensive activity of garlic. Many garlic-based products are present on the market now. They can be classified into four groups, that is, garlic essential oil, garlic oil macerate, garlic powder and garlic extract. Compared with other garlic preparations, dehydrated garlic powder is thought to retain the same ingredients as raw garlic, both water-soluble and organic-soluble, although the proportions and amounts of various constituents may differ significantly. ${ }^{35,36}$ Both Allicor and Kwai contain just garlic powder, but Allicor is a time-released preparation; its biological effect lasts for $12-16 \mathrm{~h}$ after administration of a single dose. ${ }^{37}$ DBP lowering in Allicor-treated participants may be explained by the presence of remnants of bioactive compounds in the circulation. The difference between Kwai and Allicor in their biological effects may be due to slower disintegration of Allicor tablets during digestion. This process results in the absorption of bioactive compounds in a dispensed manner, providing low and steady concentrations of active metabolites to the circulation. The different effects of Allicor and Kwai on DBP may be due to prolonged action of Allicor, which may allow better bioavailability of the vasoactive constituents of garlic powder.

Hypertension is an acknowledged major risk factor for cardiovascular disease and death in both men and women. Despite a historical 
focus by clinicians on the importance of DBP risks, epidemiological data from numerous large-scale studies have clearly shown that both SBP and DBP are important determinants of cardiovascular risk, even in mild and moderate arterial hypertension. ${ }^{8,38,39}$ In addition, even high-normal blood pressure is associated with an increased risk of cardiovascular disease. ${ }^{40}$ It is generally thought that rigid normalization of blood pressure may prolong life and reduce the cardiovascular sequelae of hypertension, possibly including coronary heart disease. ${ }^{41}$ Thus, the moderate and statistically significant hypotensive effect of the time-released garlic-based dietary supplement, Allicor, may provide considerable benefits for the dietary prevention of cardiovascular diseases.

\section{ACKNOWLEDGEMENTS}

This work was supported by a grant from the Institute for Atherosclerosis Research (Moscow, Russia).

1 Bordia AK, Joshi HK, Sanadhya YK, Bhu N. Effect of essential oil of garlic on serum fibrinolytic activity in patients with coronary artery disease. Atherosclerosis 1977; 28: $155-159$.

2 Lau B, Lam F, Wang-Chen R. Effect of odor-modified garlic preparation on blood lipids. Nutr Res 1987; 7: 139-149.

3 Harenberg J, Giese C, Zimmermann R. Effect of dried garlic on blood coagulation, fibrinolysis, platelet aggregation and serum cholesterol levels in patients with hyperlipoproteinemia. Atherosclerosis 1988; 74: 247-249.

4 Zimmermann W, Zimmermann B. Reduction in elevated blood lipids in hospitalised patients by a standardised garlic preparation. Br J Clin Pract 1990; 69: S20-S23.

5 Phelps S, Harris WS. Garlic supplementation and lipoprotein oxidation susceptibility. Lipids 1993; 28: 475-477.

6 Campbell JH, Efendy JL, Smith NJ, Campbell GR. Molecular basis by which garlic suppresses atherosclerosis. J Nutr 2001; 131: 1006-1009.

7 Kannel WB. Framingham study insights into hypertensive risk of cardiovascular disease. Hypertens Res 1995; 18: 181-196.

8 Donnell CJ, Kannel WB. Cardiovascular risks of hypertension: lessons from observational studies. J Hypertens 1998; 16: S3-S7.

9 Ernst E. Cardiovascular effects of garlic (Allium sativum): a review. Pharmatherapeutica 1987; 5: 83-89.

10 Auer W, Eiber A, Hertkorn E, Hoehfeld E, Koehrle U, Lorenz A, Mader F, Merx W, Otto G, Schmid-Otto B, Taubenheim H. Hypertension and hyperlipidaemia: garlic helps in mild cases. Br J Clin Pract 1990; 69: S3-S6.

11 Silagy CA, Neil HA. A meta-analysis of the effect of garlic on blood pressure. J Hypertens 1994; 12: 463-468.

12 Neil HA, Silagy CA, Lancaster T, Hodgeman J, Vos K, Moore JW, Jones L, Cahill J, Fowler GH. Garlic powder in the treatment of moderate hyperlipidaemia: a controlled trial and meta-analysis. J R Coll Physicians Lond 1996; 30: 329-334.

13 Steiner M, Khan AH, Holbert D, Lin RI. A double-blind crossover study in moderately hypercholesterolemic men that compared the effect of aged garlic extract and placebo administration on blood lipids. Am J Clin Nutr 1996; 64: 866-870.

14 McMahon FG, Vargas R. Can garlic lower blood pressure? A pilot study. Pharmacotherapy 1993; 13: 406-407.

15 Foushee DB, Ruffin J, Banerjee U. Garlic as a natural agent for the treatment of hypertension: a preliminary report. Cytobios 1982; 34: 145-152.

16 Mayeux PR, Agrawal KC, Tou JS, King BT, Lippton HL, Hyman AL, Kadowitz PJ, McNamara DB. The pharmacological effects of allicin, a constituent of garlic oil. Agents Actions 1988; 25: 182-190.
17 Martin N, Bardisa L, Pantoja CV, Roman R, Vargas M. Experimental cardiovascular depressant effects of garlic (Allium sativum) dialysate. J Ethnopharmacol 1992; 37: 145-149.

18 Kaye AD, Nossaman BD, Ibrahim IN, Feng CJ, McNamara DB, Agrawal KC, Kadowitz $\mathrm{PJ}$. Analysis of responses of allicin, a compound from garlic, in the pulmonary vascular bed of the cat and in the rat. Eur J Pharmacol 1995; 276: 21-26.

19 Al Qattan KK, Alnaqeeb MA, Ali M. The antihypertensive effect of garlic (Allium sativum) in the rat two-kidney—one-clip Goldblatt model. J Ethnopharmacol 1999; 66: 217-222.

20 Al Qattan KK, Khan I, Alnaqeeb MA, Ali M. Thromboxane-B2, prostaglandin-E2 and hypertension in the rat 2-kidney 1-clip model: a possible mechanism of the garlic induced hypotension. Prostaglandins Leukot Essent Fatty Acids 2001; 64: 5-10.

21 Brandle M, Al Makdessi S, Weber RK, Dietz K, Jacob R. Prolongation of life span in hypertensive rats by dietary interventions. Effects of garlic and linseed oil. Basic Res Cardiol 1997; 92: 223-232.

22 Elkayam A, Mirelman D, Peleg E, Wilchek M, Miron T, Rabinkov A, Sadetzki S, Rosenthal T. The effects of allicin and enalapril in fructose-induced hyperinsulinemic hyperlipidemic hypertensive rats. Am J Hypertens 2001; 14: 377-381.

23 Fallon MB, Abrams GA, Abdel-Razek TT, Dai J, Chen SJ, Chen YF, Luo B, Oparil S, Ku DD. Garlic prevents hypoxic pulmonary hypertension in rats. Am J Physiol 1998; 275: 283-287.

24 Pedraza Chaverri J, Tapia E, Medina Campos ON, de los Angeles Granados M, Franco M. Garlic prevents hypertension induced by chronic inhibition of nitric oxide synthesis. Life Sci 1998; 62: 71-77.

25 Mohamadi A, Jarrell ST, Shi SJ, Andrawis NS, Myers A, Clouatre D, Preuss HG. Effects of wild versus cultivated garlic on blood pressure and other parameters in hypertensive rats. Heart Dis 2000; 2: 3-9.

26 Preuss HG, Clouatre D, Mohamadi A, Jarrell ST. Wild garlic has a greater effect than regular garlic on blood pressure and blood chemistries of rats. Int Urol Nephrol 2001; 32: $525-530$.

27 Sial AY, Ahmad SI. Study of the hypotensive action of garlic extract in experimental animals. J Pak Med Assoc 1982; 32: 237-239.

28 Aqel MB, Gharaibah MN, Salhab AS. Direct relaxant effects of garlic juice on smooth and cardiac muscles. J Ethnopharmacol 1991; 33: 13-19.

29 Pantoja CV, Chiang LC, Norris BC, Concha JB. Diuretic, natriuretic and hypotensive effects produced by Allium sativum (garlic) in anaesthetized dogs. J Ethnopharmacol 1991; 31: 325-331.

30 Pantoja CV, Norris BC, Contreras CM. Diuretic and natriuretic effects of chromatographically purified fraction of garlic (Allium sativum). J Ethnopharmacol 1996; 52: 101-105.

31 Pantoja CV, Martin NT, Norris BC, Contreras CM. Purification and bioassays of a diuretic and natriuretic fraction from garlic (Allium sativum). J Ethnopharmacol 2000; 70: 35-40.

32 Siegel G, Walter A, Schnalke F, Schmidt A, Buddecke E, Loirand G, Stock G. Potassium channel activation, hyperpolarization, and vascular relaxation. Z Kardiol 1991; 80: S9-S24.

33 Siegel G, Emden J, Wenzel K, Mironneau J, Stock G. Potassium channel activation in vascular smooth muscle. Adv Exp Med Biol 1992; 311: 53-72.

34 Block E. The chemistry of garlic and onions. Sci Am 1985; 252: 114-119.

35 Iberl B, Winkler G, Mueller B, Knobloch K. Quantitative determination of allicin and alliin from garlic by HPLC. Planta Med 1990; 56: 320-326.

36 Amagase $\mathrm{H}$, Petesch BL, Matsuura H, Kasuga S, Itakura Y. Intake of garlic and its bioactive components. J Nutr 2001; 131: S955-S962.

37 Orekhov AN, Tertov VV, Sobenin IA, Pivovarova EM. Direct anti-atherosclerosis-related effects of garlic. Ann Med 1995; 27: 63-65.

38 Kannel WB. Review of recent Framingham study hypertension research. Curr Hypertens Rep 2000; 2: 239-240.

39 Kato J, Aihara A, Kikuya M, Matsubara M, Ohta M, Ohkubo T, Tsuji I, Sekino H, Meguro T, Imai Y. Risk factors and predictors of coronary arterial lesions in Japanese hypertensive patients. Hypertens Res 2001; 24: 3-11.

40 Vasan RS, Larson MG, Leip EP, Evans JC, O'Donnell CJ, Kannel WB, Levy D. Impact of high-normal blood pressure on the risk of cardiovascular disease. N Engl J Med 2001; 345: 1291-1297.

41 Kannel WB. Mild hypertension as a cardiovascular risk factor. Compr Ther 1981; 7: 7-14. 